\title{
Urodynamics in Women from Menopause to Oldest Age: What Motive? What Diagnosis?
}

\author{
Françoise A. Valentini, Gilberte Robain, Brigitte G. Marti \\ ER6 - Universite Pierre et Marie Curie (FAV, GR), Paris, France and Department of Physical Medicine \\ and Rehabilitation (FAV, GR, BGM), Hopital Charles Foix, Ivry-sur-Seine, France
}

\begin{abstract}
Purpose: To analyze age-associated changes as a motive for urodynamics and urodynamic diagnosis in community-dwelling menopausal women and to discuss the role of menopause and ageing.

Materials and Methods: Four hundred and forty nine consecutive menopausal women referred for urodynamic evaluation of lower urinary tract (LUT) symptoms, met the inclusion criteria and were stratified into 3 age groups: 55-64 years (A), 65-74 years (B), and 75-93 years (C). Comprehensive assessment included previous medical history and clinical examination. Studied items were motive for urodynamics, results of uroflows (free flow and intubated flow) and cystometry, urethral pressure profilometry, and final urodynamic diagnosis.

Results: The main motive was incontinence $(66.3 \%)$ with significant increase of mixed incontinence in group $\mathrm{C}(\mathrm{p}=0.028)$. Detrusor function significantly deteriorated in the oldest group, mainly in absence of neurological disease (overactivity $p$ $=0.019$; impaired contractility $\mathrm{p}=0.028)$. In the entire population, underactivity predominated in group $\mathrm{C}(\mathrm{p}=0.0024)$. A progressive decrease of maximum urethral closure pressure occurred with ageing. In subjects with no detrusor overactivity there was a decrease with age of detrusor pressure at opening and at maximum flow, and of maximum flow while post void residual increased only in the $\mathrm{C}$ group.

Conclusion: In our population of community-dwelling menopausal women, incontinence was the main motive for urodynamics increasing with ageing. A brisk change in LUT function of women older than 75 years underlined deterioration in bladder function with a high incidence of detrusor hyperactivity with or without impaired contractility while change in urethral function was progressive. Effect of ageing appears to be predominant compared to menopause.
\end{abstract}

Key words: ageing; women; LUTS; urodynamics

Int Braz J Urol. 2011; 37: 100-7

\section{INTRODUCTION}

Lower urinary tract (LUT) dysfunction is a major cause of reduced quality of life in the ageing population. For women the postmenopausal period has significantly increased and is now up to one third or more of the total lifespan. LUT function is affected by estrogen withdrawal and ageing. A major consequence of estrogen withdrawal is urogenital atrophy with possible contribution to urinary symptoms such as frequency, urgency and incontinence (1). Ageing is associated with a progressive decrease in autonomic innervation and of detrusor contractility $(2,3)$. Some studies have focused on identifying the effects of menopause and ageing (4-6) on lifestyle. In spite of a reported significant trend for increased prevalence of symptoms with ageing, none of these studies have identified causes. 
Despite the fact that many patients avoid discussing their problems, postmenopausal women constitute a large population who undergo urodynamics for lower urinary tract symptoms (LUTS) and urodynamics is considered as the best tool for the evaluation of patients with LUTS.

Some authors (7) have concluded that female bladder and urethral function deteriorate throughout adult life, whether or not detrusor overactivity (DO) is present. In a previous study (8) we analyzed the motive for urodynamics and the urodynamic diagnosis in a population of community-dwelling elderly females (80+ years); we showed that incontinence was the main motive and DO the main diagnosis.

Our objectives in this study were to extend our analysis to a large population of postmenopausal community-dwelling women referred as outpatients for evaluation of LUTS and to consider the relationships between menopause and ageing on the changes in the motive of referral and in LUT function.

\section{MATERIALS AND METHODS}

The population consisted of 449 consecutive women, community-dwelling, aged $\geq 55$ years who underwent urodynamics for LUTS in our outpatient urodynamics clinic between January 2005 and March 2008. Patients were stratified in 3 age groups: 55-64 years (A, short-term menopause), 65-74 years (B, middle-term menopause), and 75-93 years ( $\mathrm{C}$, longterm menopause). The lowest age group (55 years) was set so that the women were all postmenopausal (in France, the mean age of menopause is 50.1 years). This retrospective study was conducted in accordance with the declaration of Helsinki. The local practice of our Ethics Committee does not require a formal institutional review board approval for retrospective studies.

Urodynamics investigations were performed according to Good Urodynamic Practices (GUP) (9) using the Laborie's Dorado ${ }^{\circledR}$ unit. Detailed urodynamic session included one initial free uroflow (FF1), cystometry and pressure-flow study (PFs) in a seated position, urethral pressure profilometry (UPP) in supine position, bladder empty before cystometry and bladder filled (according with the functional bladder capacity) after PFs and then a second FF. Cystometry was performed with a 7F triple-lumen urethral catheter. Bladder was filled with saline at room-temperature at a medium filling rate of $50 \mathrm{~mL} / \mathrm{min}$. Abdominal pressure was recorded using a punctured intra-rectal balloon catheter.

Pressures were zeroed to atmosphere with the transducers placed at the level of the upper edge of the symphisis pubis.

No routine provocative manoeuvres for DO were performed but according to GUP coughs were used as quality control of pressure recordings (9).

All patients had an evaluation including medical history and usual medication, bladder diary for at least 48 hours including voiding times and voided volumes during day and night-time, physical examination and dipstick urinalysis.

Specific evaluation comprised of a history of LUTS, previous history of neurological disease (stroke, multiple sclerosis, lumbar injury, etc.) or dementia, pelvic floor status and previous pelvic surgery. Patients with LUTS due to a specific physiopathology (complete spinal cord injury) were excluded, as well as those who were unable to perform the standardized protocol for complete retention (no FF and PFs were possible) or severe dementia (involving failure to understand simple orders or Mini Mental State < 20).

Two physicians independently assessed urodynamics; good agreement occurred in up to $95 \%$ of the files. In the remaining 5\%, a third interpretation was carried out jointly to agree on a single conclusion. Studied items were motive for urodynamics, feasibility of uroflows (FF and PFs), detrusor behavior during filling cystometry, UPP and final urodynamic diagnosis. Feasibility of uroflows was defined by a voided volume higher than $100 \mathrm{~mL}$. To analyze the detrusor behavior during filling cystometry, an additive stratification was used with the following parameters: age $(A, B, C)$, without neurological disease (I) or with (II) (was verified that previous pelvic surgery did not lead to significant difference).

Quality of life was assessed using the ICIQ-UI-SF questionnaire for incontinent patients (10) and visual analog scale (VAS) for continent patients. 


\section{Statistical Analysis}

Data are presented as mean $\pm \mathrm{SD}$ and range. The Wilcoxon signed rank test was used for comparison of related samples, analysis of variance and the chi-square test to compare unrelated samples. Statistical analysis was performed using SAS, version 5.0 (SAS Institute, Inc., Cary, NC). All statistical results were considered significant at $\mathrm{p}<0.05$.

\section{RESULTS}

\section{Population}

The 3 sub-groups were homogeneous in terms of numbers of patients: $\mathrm{A}=137, \mathrm{~B}=155, \mathrm{C}=157$. Mean age was $59 \pm 3$ years in A, $70 \pm 3 y$ in $B$ and 81 \pm 4 years in $\mathrm{C}$.

Oral or transdermal hormone replacement therapy (HRT) was respectively taken by $11(8.0 \%)$ women in A and $29(18.8 \%)$ in B; in C, 15 women $(9.6 \%)$ received estrogen locally.

\section{Motive for Urodynamics}

Table-1 lists the motive for urodynamics by age-groups. Incontinence was the main motive, evoked by 298 (66.3\%) patients. Mixed incontinence increased with ageing with a significant difference between groups A and C ( $p=0.028)$.

ICIQ-SF score (maximum 21) and VAS score (maximum 10) are detailed in Table-2.

\section{Previous History}

Previous history of medical disease or/and pelvic surgery was obtained by detailed questioning, and is listed in Table-3.

One hundred and sixteen (25.8\%) patients had a previous history of neurological disease $(\mathrm{A}=$ $35 ; \mathrm{B}=36 ; \mathrm{C}=45)$ and $151(33.6 \%)$ had undergone previous pelvic surgery $(A=56 ; B=54 ; C=41)$.

Eleven patients were referred for pre-operative evaluation of pelvic organ prolapse (POP); POP grade 2-3 was revealed during urogenital examination in 31 additional patients $(\mathrm{A}=9 ; \mathrm{B}=13 ; \mathrm{C}=9)$.

\section{Feasibility of the Tests}

The percentage of interpretable initial $\mathrm{FF}$ was significantly higher in age-group $\mathrm{B}(\mathrm{A}=57.8 \%$; $\mathrm{B}=$ $69.3 \% ; \mathrm{C}=47.5 \%$ ) while there was not a significant difference between the age groups for both interpretable PFs $(\mathrm{A}=64.2 \% ; \mathrm{B}=56.1 \% ; \mathrm{C}=56.4 \%)$ and $\mathrm{FF}$ at end of the session $(\mathrm{A}=94.6 \% ; \mathrm{B}=94.8 \% ; \mathrm{C}=$ $97.2 \%)$.

Table 1 - Motive for urodynamics. $P O P=$ pelvic organ prolapse.

\begin{tabular}{lccccc}
\hline Complaint & & A (55-64 years $)$ & B (65-74 years) & C ( $\geq 75$ years $)$ & Total \\
\hline & Stress & 20 & 28 & 21 & 122 \\
Incontinence & Urge & 41 & 42 & 39 & 122 \\
& Mixed & 23 & 36 & 48 & 107 \\
Number of incontinent & & $84(61.3 \%)$ & $106(68.4 \%)$ & $108(68.8 \%)$ & 298 \\
Frequency & 22 & 26 & 18 & 66 \\
Dysuria & 8 & 8 & 6 & 22 \\
Chronic incomplete retention & 3 & 6 & 14 & 23 \\
Pre-operative (POP) & 3 & 6 & 2 & 11 \\
Various (pain, infection, etc.) & & 3 & 3 & 9 & 29 \\
Number of continent & 53 & 49 & 49 & 151 \\
\hline
\end{tabular}


Table 2 - Quality of life in the studied population. ICIQ-UI-SF = International Consultation on Incontinence Questionnaire on Urinary Incontinence Short Form; VAS = Visual Analog Scale.

\begin{tabular}{lcccc}
\hline & A (55-64 years $)$ & B $(65-74$ years $)$ & $\mathbf{C}(\geq 75$ years $)$ & $\mathbf{p ~ V a l u e ~}$ \\
\hline ICIQ-UI-SF & 12.8 & 10.9 & 11.0 & n.s. \\
VAS & 5.1 & 5.0 & 4.5 & n.s. \\
\hline
\end{tabular}

n.s. $=$ not significant.

Table 3 - Previous medical history of the studied population.

\begin{tabular}{lccc}
\hline Previous History & A (55-64 years) & B (65-74 years) & C ( $\geq 75$ years $)$ \\
\hline Neurological disease & 35 & 36 & 45 \\
Cognitive impairment (Mini Mental State $\geq 20)$ & 5 & 0 & 19 \\
Pelvic surgery & & 5 & 3 \\
$\quad$ Pelvic organ prolapse & 5 & 17 & 13 \\
Hysterectomy & 17 & 29 & 19 \\
TVT or sling & 23 & 3 & 6 \\
$\quad 11$ & & 69 \\
Cure of incontinence & & 45 & 83 \\
Musculo-skeletal & 29 & 77 & 24 \\
Cardio-vascular & 13 & 16 & 15 \\
Depression & 24 & 22 & \\
Diabetes mellitus & 13 & & \\
\hline
\end{tabular}

$T V T=$ tension free vaginal tape.

\section{Cystometry (Table-4)}

Detrusor overactivity (involuntary detrusor contraction during the filling phase, DO) (9-11) and its subset detrusor hyperactivity with impaired contractility (DHIC) (12) were found in a total of 142 patients (32\%) with a significant increase in group C: $43 \%$ vs. $23 \%$ A \& $30 \%$ B ( $p=0.0004)$; detrusor underactivity (impaired detrusor contraction leading to prolonged voiding time and high residual volume) (DUA) (9) was found in 62 patients (14\%) with also a significant increase in $\mathrm{C}(\mathrm{p}=0.0024)$. Normal detrusor behavior significantly decreased in $\mathrm{C}(\mathrm{p}=$ $0.005)$.

In sub-group I (333 patients without neurological disease) the detrusor behavior was normal in $194(58.2 \%)$ with only a significant difference between subgroups I-B and I-C $(p=0.044)$. DO and
DHIC significantly increased in subgroup I-C (respectively $p=0.019$ and 0.028 ) but the increase in DUA was not significant.

In subgroup II (116 patients with neurological disease) the detrusor behavior was normal in 33 (28.4\%). DO was found in $46(39.6 \%)$, DHIC in 15 (12.9\%) and DUA in 20 (17.2\%). There was no significant difference between the 3 age groups regarding DO: II-A (45.7\%), II-B (36.1\%) and II-C (37.7\%). An increase in DHIC and DUA in subgroup II-C was observed.

Functional bladder capacity (FBC) did not change significantly with age but depended on DO (Table-5). Some other voiding parameters were modified in DO patients: $\mathrm{p}_{\text {det.op }}$ and $\mathrm{p}_{\text {det.Qmax }}$ increased, the voiding time decreased, except in group $\mathrm{A}$, and PVR was lower in group C. In the DO population, $\mathrm{p}_{\text {det.op }}$ was higher than $\mathrm{p}_{\text {det.Qmax }}$. 
Table 4 - Changes in uroflow parameters comparing free uroflow (FF) and pressure-flow study (PFs).

\begin{tabular}{lccccccccc}
\hline & \multicolumn{2}{c}{$\mathbf{A}(55-64 \mathrm{y})$} & $\mathbf{p}$ & \multicolumn{2}{c}{$\mathbf{B}(65-74 \mathrm{y})$} & $\mathbf{p}$ & \multicolumn{2}{c}{$\mathbf{C}(\geq 75 \mathrm{y})$} & $\mathbf{p}$ \\
& $\mathbf{F F 1}$ & $\mathbf{P F s}$ & & $\mathbf{F F 1}$ & $\mathbf{P F s}$ & & $\mathbf{F F 1}$ & PFs & \\
\hline $\mathrm{Q}_{\text {max }}(\mathrm{mL} / \mathrm{s})$ & $23 \pm 11$ & $14 \pm 8$ & $<0.0001$ & $19 \pm 11$ & $14 \pm 7$ & $<0.0001$ & $13 \pm 8$ & $10 \pm 5$ & 0.0358 \\
$\mathrm{~V}_{\text {oided }}(\mathrm{mL})$ & $256 \pm 154$ & $320 \pm 159$ & 0.007 & $249 \pm 150$ & $333 \pm 147$ & 0.0008 & $124 \pm 122$ & $233 \pm 143$ & $<0.0001$ \\
$\mathrm{PVR}(\mathrm{mL})$ & $34 \pm 74$ & $77 \pm 117$ & 0.007 & $59 \pm 96$ & $101 \pm 142$ & 0.0004 & $74 \pm 121$ & $129 \pm 143$ & $<0.0001$ \\
$\mathrm{t}_{\text {void }}(\mathrm{s})$ & $27 \pm 19$ & $64 \pm 48$ & $<0.0001$ & $34 \pm 26$ & $66 \pm 42$ & $<0.0001$ & $27 \pm 25$ & $61 \pm 49$ & $<0.0001$ \\
\hline
\end{tabular}

\section{Uroflow Parameters (Table-6)}

Maximum flow rate diminished with age whether measured during FF or PFs, and was significantly lower during PFs. The decrease was independent of DO. Post void residual (PVR) significantly increased only in group $\mathrm{C}$.

\section{UPP (Table-7)}

Maximum urethral closure pressure (MUCP) decreased steadily with age remaining in the range of the "theoretical" value which is bladder filled (110 - age) $\pm 20 \%$ in $\mathrm{cm} \mathrm{H}_{2} \mathrm{O}$ (13).

MUCP was non significantly higher in continent patients either with bladder empty or bladder filled, but was significantly lower bladder filled vs. bladder empty in incontinent whatever the age and incontinent in group B.

\section{Urodynamic Diagnosis}

Detrusor hyperactivity (DO or DHIC) was the main urodynamic diagnosis increasing signifi- cantly in group C: $68 / 157$ (43\%) vs. $38 / 137$ (27\%) (A) and 36/155 (23\%) (B) (p=0.0004) whatever the neurological status. DO was significantly more frequent $(\mathrm{p}=0.007)$ in the age-group C $(28 \%)$ vs. A (23\%) and B (15\%) and DHIC increased with ageing: $4 \%$ in $A, 8 \%$ in $B$ and $15 \%$ in $C$.

DUA was predominant in the oldest group $34 / 157$ (22\%) compared with 14/137 (10\%) (A) and $14 / 155$ (9\%) (B). That dysfunction was associated with an incompetent sphincter in 2 A patients, $5 \mathrm{~B}$ and $14 \mathrm{C}$.

Intrinsic sphincter deficiency (ISD) was predominant in B 56/155 (36\%) vs. A 34/137 (25\%) or C $38 / 157(24 \%)$.

Low bladder compliance $\left(\leq 20 \mathrm{~mL} / \mathrm{cm} \mathrm{H}_{2} \mathrm{O}\right)$ was predominant in group B: 10 women of which 9 were without neurological disease.

Normal urodynamic tests were observed in $31(22 \%)$ A patients, 24 (15\%) in B and $18(11 \%)$ in C.

Various or uncertain diagnoses were more frequent in $\mathrm{A}(16 \%)$ and $\mathrm{B}(14 \%)$ than in $\mathrm{C}$ $(6 \%)$.

Table 5 -Detrusor behavior vs. age and neurological status. DO = detrusor overactivity; DHIC = detrusor hyperactivity with impaired contractility; $D U A=$ detrusor underactivity; $L C=$ low bladder compliance; $N=$ normal.

\begin{tabular}{lccccccccccccccr}
\hline & \multicolumn{3}{c}{ DO } & \multicolumn{1}{c}{ DHIC } & \multicolumn{1}{c}{ DUA } & \multicolumn{1}{c}{ LC } & \multicolumn{3}{c}{$\mathbf{N}$} \\
& A & B & C & A & B & C & A & B & C & A & B & C & A & B & C \\
\hline I without neurological disease & 16 & 10 & 27 & 3 & 10 & 15 & 10 & 10 & 22 & 5 & 9 & 2 & 69 & 79 & 46 \\
II with neurological disease & 16 & 13 & 17 & 3 & 3 & 9 & 4 & 4 & 12 & 0 & 1 & 1 & 12 & 15 & 6 \\
Number of patients & 32 & 23 & 44 & 6 & 13 & 24 & 14 & 14 & 34 & 5 & 10 & 3 & 81 & 94 & 52 \\
\hline
\end{tabular}


Urodynamics in Women from Menopause to Oldest Age

Table 6 - Influence of detrusor overactivity (DO) on the urodynamic parameters during pressure-flow study (PFs).

\begin{tabular}{lccccccccc}
\hline & \multicolumn{3}{c}{ A (55-64 years) } & \multicolumn{3}{c}{ B (65-74 years) } & \multicolumn{3}{c}{ C ( $\geq 75$ years $)$} \\
& DO & no DO & p & DO & no DO & p & DO & no DO & p \\
\hline FBC $(\mathrm{mL})$ & $288 \pm 119$ & $407 \pm 123$ & $<0.0001$ & $314 \pm 124$ & $436 \pm 125$ & 0.0005 & $289 \pm 128$ & $427 \pm 128$ & $<0.0001$ \\
$\mathrm{~V}_{\text {voided }}(\mathrm{mL})$ & $237 \pm 119$ & $353 \pm 154$ & 0.0004 & $231 \pm 101$ & $362 \pm 145$ & 0.0004 & $198 \pm 130$ & $274 \pm 147$ & 0.0074 \\
$\mathrm{Q}_{\max }(\mathrm{mL} / \mathrm{s})$ & $13 \pm 7$ & $15 \pm 8$ & n.s. & $12 \pm 6$ & $15 \pm 8$ & n.s. & $10 \pm 5$ & $11 \pm 6$ & n.s. \\
$\mathrm{PVR}(\mathrm{mL})$ & $71 \pm 98$ & $80 \pm 124$ & n.s. & $119 \pm 111$ & $96 \pm 149$ & n.s. & $87 \pm 88$ & $175 \pm 174$ & 0.015 \\
$\mathrm{p}_{\text {det.op }}$ & $29 \pm 20$ & $17 \pm 14$ & 0.0012 & $30 \pm 24$ & $14 \pm 12$ & 0.0022 & $26 \pm 18$ & $13 \pm 16$ & $<0.001$ \\
$\left(\mathrm{~cm} \mathrm{H} \mathrm{H}_{2} \mathrm{O}\right)$ & & & & & & & & & \\
$\mathrm{p}_{\text {det.(.max }}$ & $27 \pm 11$ & $23 \pm 13$ & n.s. & $29 \pm 19$ & $21 \pm 14$ & 0.0032 & $24 \pm 14$ & $15 \pm 13$ & 0.0011 \\
$\left(\mathrm{~cm} \mathrm{H} \mathrm{H}_{2} \mathrm{O}\right)$ & & & & & & & & & \\
$\mathrm{t}_{\text {void }}(\mathrm{s})$ & $66 \pm 67$ & $63 \pm 38$ & n.s. & $49 \pm 25$ & $70 \pm 45$ & n.s. & $49 \pm 36$ & $73 \pm 57$ & n.s. \\
\hline
\end{tabular}

n.s. $=$ not significant.

Table 7 - Maximal urethral closure pressure (MUCP) vs. age and continence status.

\begin{tabular}{|c|c|c|c|c|c|c|}
\hline \multirow[t]{2}{*}{ MUCP cm $\mathrm{H}_{2} \mathrm{O}$} & \multicolumn{2}{|c|}{ A $(55-64$ years $)$} & \multicolumn{2}{|c|}{ B (65-74 years) } & \multicolumn{2}{|c|}{$\mathrm{C}(\geq 75$ years $)$} \\
\hline & $\begin{array}{c}\text { continent } \\
70 \pm 28\end{array}$ & $\begin{array}{c}\text { incontinent } \\
62 \pm 26\end{array}$ & $\begin{array}{c}\text { continent } \\
56 \pm 24\end{array}$ & $\begin{array}{c}\text { incontinent } \\
52 \pm 23\end{array}$ & $\begin{array}{c}\text { continent } \\
50 \pm 27\end{array}$ & $\begin{array}{c}\text { incontinent } \\
43 \pm 18\end{array}$ \\
\hline Bladder filled & $62 \pm 28$ & $55 \pm 28$ & $45 \pm 24$ & $44 \pm 22$ & $39 \pm 21$ & $35 \pm 18$ \\
\hline $\mathrm{p}$ & 0.07 & 0.0008 & 0.003 & 0.0002 & 0.07 & $<0.0001$ \\
\hline "theoretical" value bladder filled & \multicolumn{2}{|c|}{$38-62$} & \multicolumn{2}{|c|}{$30-50$} & \multicolumn{2}{|c|}{$24-36$} \\
\hline
\end{tabular}

\section{COMMENTS}

This study was retrospective and therefore has its own limitations. However, we used a standardized protocol for urodynamics and all files contain the same items. The population is representative of community-dwelling women with urinary disorders and age-groups are homogeneous in the number of patients.

In our postmenopausal population, urinary incontinence (UI) was the main motive for urodynamics with a percentage variation between 61.3 and 68.8. These values, above the estimated prevalence of urinary incontinence in middle-aged and older women (4), resulted from our recruitment, i.e. women referred for evaluation of LUT dysfunction. In the general population many patients avoid discussing problems related to incontinence, lowering the recorded per- centages. Prevalence of urinary incontinence during the menopausal transition has been reported with a variation from $8 \%$ to $56 \%$; nevertheless, evidence that menopause is an independent factor in the prevalence of incontinence remains lacking (4).

Mixed UI increases with ageing, probably due to the association of a decreased urethral sphincter function and occurrence of detrusor overactivity.

Urge incontinence does not vary significantly with age. On the other hand, stress incontinence appears as slightly predominant in age-group B in patients who take oral or transdermal HRT (or who have taken it five to ten years after menopause); this is consistent with the findings of Steinauer et al. (14) who report an increased risk for stress incontinence in women taking HRT.

Quality of life scores show that the impact of LUT dysfunction remains stable with age. 
Achievement of interpretable tests, requiring a "comfortable" environment and some relaxation is often difficult at the beginning of the session.

As previously reported (7) FBC does not diminish with age and is smaller, with no variation with age, in DO subjects.

There are some surprising results as the subgroup B differs from the others in terms of normal detrusor behavior, low occurrence of DO and low bladder compliance. One plausible explanation may by the higher incidence of stress incontinence in this group.

DO increases with age regardless of a history of neurological disease or previous pelvic surgery. In the entire DO population, $\mathrm{p}_{\text {det.op }}$ is higher than $\mathrm{p}_{\text {det.Qmax }}$ which may imply a common finding of an incomplete sphincter relaxation at the onset of flow.

DHIC, is a common condition in frail elderly individuals (12), and DUA increases in the same way; these two latter behaviors lead respectively to UI with high PVR and to chronic retention. The decrease of detrusor contractility with ageing can be related to the decrease in caveolae, bladder weight and smooth muscle density with age (3).

For a complaint consistent with bladder overactivity (urge or mixed incontinence, frequency) similar percentage (near $85 \%$ ) in DO plus DHIC is found in the 3 age-groups. $Q_{\max }, p_{\text {det.op }}$ and $p_{\text {det.Qmax }}$ all decline with age in women without DO. This observation is consistent with previous studies which show age-associated deterioration of the detrusor contractility $(15,16)$. However, decreased contractility allows an effective emptying except in the oldest age-group where PVR increases.

MUCP decreases with age, a result consistent with previous studies (7); with the bladder filled, the values are in the range of the "theoretical values" (13). Perucchini et al. (17) reported that this decrease could be the reflect of the association with age of a loss of striated muscle in the female urethra.

In incontinent women, the decrease observed between bladder empty and bladder filled demonstrates a lack of adaptation of the urethral sphincter to bladder filling. This behavior could be the explained by both sphincter sarcopenia and impaired pelvic floor.

An unexpected and unexplained finding is the significant decrease of MUCP bladder filled in continent women of the middle age-group.
For a complaint consistent with an impaired sphincter function (stress or mixed incontinence), a lower urethral closure pressure than expected for age was found in only $52.6 \%$ (A), $57.4 \%(\mathrm{~B})$ and $62.7 \%$ (C).

Urodynamic diagnosis was found in a high percentage ( $90 \%$ or more) of the 3 age groups.

Abnormal detrusor behavior appears as the main final diagnosis with specific changes according to ageing. In the oldest women without neurological disease, a significant increase of DO, DHIC was observed; the increase of DUA was not considered significant. The incidence of DHIC and DUA in this age group is more likely related to decreased $p_{\text {det.op }}$ and $\mathrm{p}_{\text {det.Omax }}$ leading to an increased PVR.

In all groups, complaint of stress and urge incontinence was respectively associated with ISD and detrusor hyperactivity (DO or DHIC). Mixed incontinence was associated with ISD in age-groups $\mathrm{B}$ and $\mathrm{C}$ and with detrusor hyperactivity in $\mathrm{C}$.

One final question: Can the responsibility of the changes in LUT function be attributed to the normal ageing process or to menopause?

Although menopause has been shown to be associated with urinary incontinence, evidence for it being an independent factor in the prevalence of urinary incontinence and bladder dysfunction remains lacking. In our population, menopause and ageing could be considered as independent factors as the percentage of women taking HRT is insignificant. Brisk changes in LUT function occurred in the oldest group while for the two other age groups (A and B) changes were progressive. Note that the change in urethral function is progressive with ageing. Therefore, if the role of menopause can be considered in younger and middle age, we propose that the role of ageing is predominant with a strenuous expression in advanced age as it mainly implies the detrusor which is less dependent on estrogens.

\section{CONCLUSION}

In our community-dwelling population of menopausal females urinary incontinence remains the main motive for urodynamics and there is an increasing complaint of urgency with ageing. The lack 
of adaptation of the sphincter to bladder filling could explain the complaint of incontinence. The role of ageing clearly results from deterioration in bladder function leading to DO, DHIC and DUA in the oldest group. Due to the brisk changes in detrusor function between the middle and the oldest age groups the role of ageing appears to predominate. Further studies are needed to search for a better understanding of the neural control of micturition in ageing women and to better define the conditions leading to impaired detrusor function in the oldest age groups.

\section{CONFLICT OF INTEREST}

None declared.

\section{REFERENCES}

1. Cardozo L, Robinson D: Special considerations in premenopausal and postmenopausal women with symptoms of overactive bladder. Urology. 2002; 60(5 Suppl 1): 64-71; discussion 71.

2. Gosling JA: Modification of bladder structure in response to outflow obstruction and ageing. Eur Urol. 1997; 32(Suppl 1) :9-14.

3. Elbadawi A, Yalla SV, Resnick NM: Structural basis of geriatric voiding dysfunction. II. Aging detrusor: normal versus impaired contractility. J Urol. 1993; 150: $1657-67$.

4. Sherburn M, Guthrie JR, Dudley EC, O'Connell HE, Dennerstein L: Is incontinence associated with menopause? Obstet Gynecol. 2001; 98: 628-33.

5. Chen YC, Chen GD, Hu SW, Lin TL, Lin LY: Is the occurrence of storage and voiding dysfunction affected by menopausal transition or associated with the normal aging process? Menopause. 2003; 10: 203-8.

6. Pfisterer MH, Griffiths DJ, Rosenberg L, Schaefer W, Resnick NM: Parameters of bladder function in pre-, peri-, and postmenopausal continent women without detrusor overactivity. Neurourol Urodyn. 2007; 26: 356-61.

7. Pfisterer MH, Griffiths DJ, Schaefer W, Resnick NM: The effect of age on lower urinary tract function: a study in women. J Am Geriatr Soc. 2006; 54: 40512.

8. Valentini FA, Robain G, Marti BG, Nelson PP: Urodynamics in women from menopause to oldest age: what motive? what diagnosis? Int Braz J Urol. 2010; 36: 218-24.

9. Schäfer W, Abrams P, Liao L, Mattiasson A, Pesce F, Spangberg A, et al.: Good urodynamic practices: uroflowmetry, filling cystometry, and pressure-flow studies. Neurourol Urodyn. 2002; 21: 261-74.

10. Avery K, Donovan J, Peters TJ, Shaw C, Gotoh M, Abrams P: ICIQ: a brief and robust measure for evaluating the symptoms and impact of urinary incontinence. Neurourol Urodyn. 2004; 23: 322-30.

11. Abrams P, Cardozo L, Fall M, Griffiths D, Rosier P, Ulmsten $\mathrm{U}$, et al.: The standardisation of terminology of lower urinary tract function: report from the Standardisation Sub-committee of the International Continence Society. Neurourol Urodyn. 2002; 21: 167-78.

12. Resnick NM, Yalla SV: Detrusor hyperactivity with impaired contractile function. An unrecognized but common cause of incontinence in elderly patients. JAMA. 1987; 257: 3076-81.

13. Constantinou CE: Urethrometry: considerations of static, dynamic, and stability characteristics of the female urethra. Neurourol Urodyn 1988; 7: 521-39.

14. Steinauer JE, Waetjen LE, Vittinghoff E, Subak LL, Hulley SB, Grady D, et al.: Postmenopausal hormone therapy: does it cause incontinence? Obstet Gynecol. 2005; 106: 940-5.

15. van Mastrigt R: Age dependence of urinary bladder contractility. Neurourol Urodyn 1992; 11: 315-7.

16. Resnick NM, Elbadawi A, Yalla SV: Age and the lower urinary tract: what is normal? Neurourol Urodyn 1995; 14: 577-579.

17. Perucchini D, DeLancey JO, Ashton-Miller JA, Galecki A, Schaer GN: Age effects on urethral striated muscle. II. Anatomic location of muscle loss. Am J Obstet Gynecol. 2002; 186: 356-60.

Accepted after revision:

June 26, 2010 